\title{
Culture-proven disseminated Mycobacterium Bovis infection (BCG-Osis) following intravesical BCG immunotherapy in a patient with bladder carcinoma-in-situ: 'Case report'
}

\author{
Ahmed Ghonaimy ${ }^{1,4^{*}}$, Tariq Qadeer ${ }^{2}$, Sudhir Lohani ${ }^{2}$, John Schofield ${ }^{3}$ and Sanjeev Madaan ${ }^{1}$
}

\begin{abstract}
We are reporting a case of culture-proven disseminated Mycobacterium Bovis infection (BCG-Osis), involving lung, bone-marrow and urinary tract, after intravesical Bacillus Calmette-Guérin (BCG) immunotherapy for bladder carcinoma-in-situ. A 71-year-gentleman presented with fever shortly after intravesical BCG instillation. He was initially treated for presumed Urinary Tract Infection, but negative urine culture and persistent fever prompted us to consider alternative diagnoses. Empirical treatment for BCG-Osis was commenced after initial negative workup including blood culture, echocardiography, computed tomography (CT) Chest, and Kidney-Ureter-Bladder (KUB). However, he remained febrile and leukopenic, so bone-marrow examination was performed along with repeating CT Chest, which revealed non-caseating granulomas and small nonspecific pulmonary nodules, respectively, supporting our provisional diagnosis. Interestingly, Mycobacterium Bovis was finally isolated from one of his urine specimens, confirming our diagnosis.
\end{abstract}

Keywords: Bladder carcinoma-in-situ, BCG-Osis, Culture-proven, Mycobacterium Bovis, BCG immunotherapy, Case report

\section{Background}

Mycobacterium Bovis Bacillus Calmette-Guérin (BCG) immunotherapy has been used over many decades to treat bladder cancer in-situ with substantial efficacy [1, 2]. It is recommended by the European Association of Urology (EAU) for intermediate and high-risk non muscle invasive bladder cancer (NMIBC) to reduce the risk of tumour recurrence [3]. Unfortunately, disseminated BCG infection (BCG-Osis) is one of its known side effects [4], particularly more so when its instillation is complicated

\footnotetext{
*Correspondence: Ahmed.ghonaimy@nhs.net

1 Department of Urology and Nephrology, Darent Valley Hospital, Dartford, UK

Full list of author information is available at the end of the article
}

by traumatic catheterization [2]. It often proves to be a diagnostic and management dilemma, as the decision of empirical-treatment has to be made early in the course of illness while histology and culture reports are still pending and radiological evidence is largely nonspecific $[4,5]$. Later in the course of illness, supportive evidence including; the presence of granulomatous inflammation on histology and/or evidence of tuberculous DNA in the specimen, may become available. Nevertheless, it is rare for Mycobacterium Bovis culture to become positive, and thus, diagnosis remains mainly clinical [6].

We present a case of BCG-Osis possibly related to traumatic catheterization, confirmed by a positive urine culture. 


\section{Case presentation}

A 71-year-old man of British origin, who has a lifelong smoking history, got admitted with dysuria, haematuria, lethargy, rigors, and temperature of $39^{\circ} \mathrm{C}$. Further review of systems was negative for any other foci of infection. He had urinary catherization for maintenance BCG immunotherapy earlier that day in the morning with an interval of about $10 \mathrm{~h}$ between catheterization and fever onset). It is noteworthy that it was a difficult catheterization and his catheter tip had a tiny speck of blood on the first go. In view of potential infectious complications, the possibility of postponing treatment was contemplated, but the patient was keen on proceeding with BCG instillation.

His past medical history is significant for bladder carcinoma in situ (G2pTa TCC + CIS), which was diagnosed 1 -year ago. He had previously received six installations of BCG vaccine, which is a live attenuated Mycobacterium Bovis (OncoTICE BCG $12.5 \mathrm{mg}$ per vial containing $2-8 \times 10^{8}$ CFU Tice BCG), without any immediate side effects. Following this, he had rigid cystoscopy with biopsy and was found to have no evidence of recurrence of his malignancy, and was, therefore, started on maintenance BCG immunotherapy.

Two years ago, he had laparoscopic anterior resection for low rectal cancer with defunctioning loop ileostomy which was closed after one year, along with parastomal hernia repair. Surveillance CT scans and carcinoembryonic antigen (CEA) level did not show any evidence of recurrence of bowel cancer to date. His other medical problems include asymptomatic renal calculus in the right kidney, atrial fibrillation, hypertension, and hypercholesterolemia. He has had no prior history of Tuberculosis (TB) and denied any close TB contacts. Likewise, there was no evidence to suggest immunocompromised status.

Examination revealed a temperature of $39{ }^{\circ} \mathrm{C}$, Blood Pressure 112/77 $\mathrm{mmHg}$, heart rate $77 \mathrm{bpm}$, respiratory rate $20 / \mathrm{min}$, and oxygen saturation $96 \%$ in room air. $\mathrm{He}$ had normal respiratory and heart sounds.

Likewise, no signs of infective endocarditis, meningitis, or skin or joint infection were noted. Abdomen was soft and non-tender.

His urine dip was positive (Nitrate + , Leukocytes +++ , Erythrocytes +++ , Protein +++ and bilirubin +$)$. Blood tests showed White blood Cells (WBC) of $8.5 / \mathrm{mm}^{3}$, C Reactive Protein (CRP) $47 \mathrm{mg} / \mathrm{L}$. Chest $\mathrm{x}$-ray was normal. ECG showed rate-controlled atrial fibrillation. Urine culture was sent and the patient was given gentamycin for likely Urinary tract infection (UTI) based on his symptoms, urine dip and history of recent catherization. His early urine and blood culture did not show any growth.

Despite one week of empirical gentamycin for presumed UTI, he continued to spike temperature. Keeping his previous history of kidney stones in mind, a fresh CTKUB was requested, which did not show any hydronephrosis or change in size or position of the stone along with left adrenal adenoma. He subsequently had transthoracic echocardiography which did not reveal any evidence of infective endocarditis which was suspected because Streptococcus Bovis endocarditis has a wellknown association with colorectal cancer [5], CT thorax was, therefore, requested, which revealed a $12-\mathrm{mm}$ subcarinal lymph node along with sub-centimetre lymph nodes at the paratracheal region.

At this point (day 10 of his admission), the respiratory consultant suspected BCG-Osis clinically, and so, 3 successive early morning urine samples were sent for acidfast bacilli (AFB) and cultures. Although the patient was initially reluctant to take anti-tuberculosis medication (due to diagnostic uncertainty and lack of evidence in favour of tuberculosis), he agreed later on when he was counselled regarding the expected time frame required for $\mathrm{TB}$ tests outcome, and thus a potential significant delay in treatment initiation. He was commenced on anti-tuberculous treatment (ATT) comprising Isoniazid $300 \mathrm{mg}$ OD, Rifampicin $600 \mathrm{mg}$ OD, and Ethambutol $1400 \mathrm{mg}(15 \mathrm{mg} / \mathrm{kg})$ along with weekly $50 \mathrm{mg}$ pyridoxine. $\mathrm{M}$. Bovis has an innate resistance to Pyrazinamide, therefore, it was excluded from the treatment regimen [6]. This regimen is extrapolated from TB treatment evidence and previous reports on the treatment of BCG infection. However, he continued to spike a temperature and had variable leukopenia (range $2.4-8.5 \mathrm{~mm}^{3}$ ), lymphopenia (range $0.1-1 \mathrm{~mm}^{3}$ ), and neutropenia (range 1.5 to $6.9 \mathrm{~mm}^{3}$ ), with normal white blood cell morphology. This lack of improvement made the patient more frustrated.

Due to these blood changes, a haematologist opinion was sought (on day 31 of admission), who performed bone-marrow aspiration and trephine biopsy. The slides were stained with Haematoxylin and Eosin (H\&E), ZiehlNeelsen (ZN), periodic acid schiff (PAS), and Grocott's technique. Immunohistochemistry for lymphoid markers (CD3 and CD20) was also performed.

Then, a repeat CT Chest-Abdomen-Pelvis was done because of persistent pyrexia while being on the antimycobacterial regimen for more than 2-weeks. It reported focal patchy opacification in both lung bases, bilateral subpleural nodularity, with no evidence of internal abscesses. A few enlarged lymph nodes were noted in the mediastinum with the largest subcarinal node measuring $13 \mathrm{~mm}$ in the short axis. There was a sub-centimetre right hilar lymph node (see Fig. 1). It was not amenable to biopsy after MDT discussion with interventional radiology consultant.

Meanwhile, 33 days after admission, the trephine biopsy was reported, showing hypercellular bone marrow 


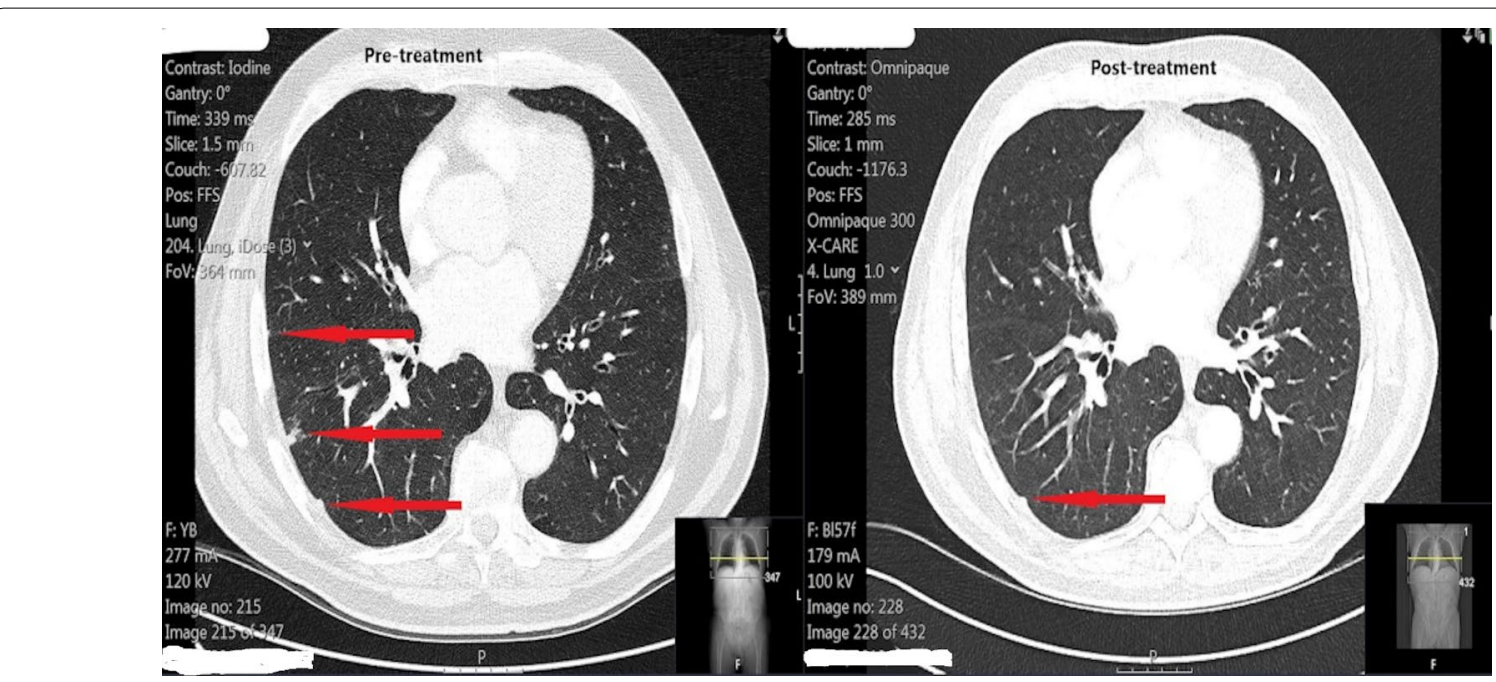

Fig. 1 Axial images of chest CT scans (left) performed before initiation of anti-tuberculosis medications, compared with Axial CT Chest image at same level on the right, 4 months after commencing treatment. There is significant improvement in subpleural nodules, as pointed by red arrows

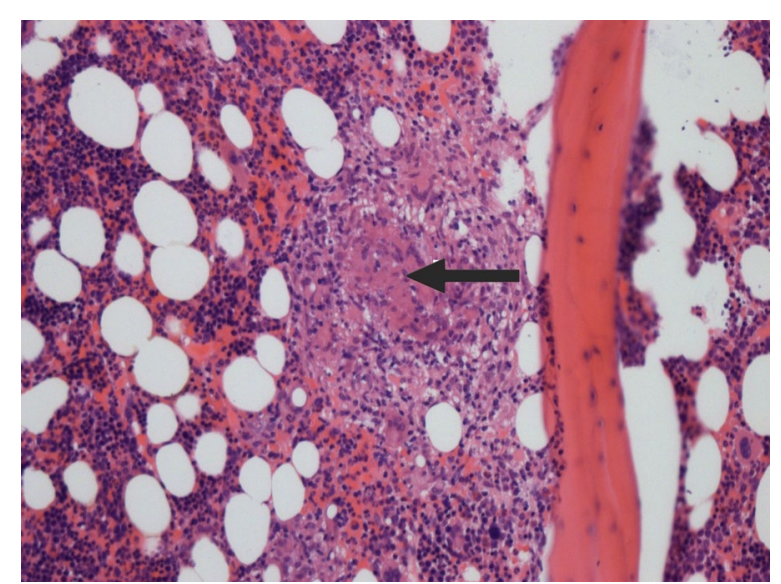

Fig. 2 Histological image (10x magnification) of bone marrow biopsy showing caseating epithelial granulomas scattered within slightly hyperplastic bone marrow (granuloma highlighted by black arrow)

with numerous non-caseating epithelial granulomata. $\mathrm{ZN}, \mathrm{PAS}$ and Grocott stains were negative. His immunoglobulin levels were normal. Possible differentials were inflammatory or infectious changes with no evidence of malignancy (see Fig. 2). However, special stains for acidfast bacilli and fungi were negative.

Finally, after a long wait of almost 82 days after admission, Mycobacterium Bovis was isolated from one of his three early morning urine specimens using BacT/ALERT ${ }^{\circledR}$ culture media (bioMérieux United Kingdom and Ireland). It was sensitive to Rifampicin, isoniazid, Ethambutol, quinolone group, but resistant to Pyrazinamide.
He became afebrile approximately 3 weeks after the start of ATT. Ethambutol was stopped after 2 months, and Rifampicin and Isoniazid were continued for another 4 months. He remained under respiratory, urology and lower GI follow-ups till completion of the treatment course which was well tolerated with no significant side effects. He is now well and apyrexial. His latest followup CT chest showed resolution of subpleural nodules (see Fig. 1) and he has been discharged from respiratory follow-up.

Urologically, his bladder was clear on recent check flexible cystoscopy. A shared decision, involving the patient and the multidisciplinary team (MDT), was made not to offer any further intravesical BCG and just continue endoscopic surveillance with the further plan of management if recurrence or progression occurs in the future.

\section{Discussion}

Although BCG immunotherapy is one of the most effective treatments for bladder carcinoma-in-situ, but it bears the risks of serious local and systemic complications $[1$, $7,8]$ since it involves instillation of live attenuated Mycobacterium Bovis in the bladder. Because of the numerous complications and their varied clinical presentation, it can provide a diagnostic and management dilemma [5, 9]. Serious side effects are encountered in $<5 \%$ of patients and can be treated effectively in almost all cases [10]. The incidence of BCG infections after BCG instillations was $1 \%$ in a register-based cohort analysis [11].

It is evident from previous studies that following BCG immunotherapy, patients mostly present acutely after intravesical BCG instillation (on the same day as in our 
case). They also can present days, months, or even years after treatment [9]. Some patients were diagnosed only by post-mortem autopsy [12].

BCG sepsis can present with fever-of-unknown-origin (FUO) [5], nonspecific symptoms of malaise, weight loss, night sweats, or organ-specific symptoms like cough, pleural effusion, urinary symptoms, or lymphadenopathy [13-15]. Late-presenting BCG disease usually involves the genitourinary tract or other localised sites, such as osteomyelitis or mycotic aneurysm [16].

One of the essential steps in management is to send tissue, urine or sputum samples for AFB staining and culture before starting treatment. But one should also be aware that M. Bovis culture is rarely positive [6] and requires a prolonged incubation of up to 8 weeks [17], thus negative culture reports should not unnecessarily change the diagnosis, particularly if the patient is responding clinically to ATT. In our case, culture was reported after approximately 11 weeks, and by that time the patient was already improving clinically.

Another important point is the timing of urine sampling for AFB culture. One should be aware that if it is done too early, i.e., within a few days of BCG instillation, the culture could be false positive from the vaccine itself as it contains live attenuated organism. In our case, it was sent off around day 10 of BCG instillation, allowing for enough bladder washout time.

CT chest findings in BCG-Osis can be very nonspecific [13], especially early in the disease process. However, later on, lymphadenopathy, consolidation, nodules, pleural effusion, or specific like miliary shadows may appear $[6,18]$. It was interesting to learn that repeating a chest CT scan was a sensible idea, as it probably provided sufficient time for the initial bacteremia to produce radiologically detectable Inflammatory changes. Although it can be argued that Bronchoalveolar lavage (BAL) or lung biopsy should have been done, but actually our patient started responding to ATT at that point and sub-pleural nodules and lymph nodes were too small to take a biopsy from them.

In most cases, treatment should be initiated based on high clinical suspicions; however, the presence of supporting evidence (like granulomatous inflammation [19], or confirming tuberculous DNA on PCR-based essay [20]) can be extremely helpful as it can enhance compliance to treatment.

Involvement of the patients in shared decision-making is of paramount importance. They should be counselled about BCG-Osis as a known complication in about $1 / 15,000$ instilled patients [2] before embarking on BCG immunotherapy. Moreover, BCG immunotherapy should be deferred in case of traumatic catherization. Although literature does not suggest any specific duration, but
1-2 weeks delay should be enough time for the mucosa to heal from minor trauma [9]. It is worth mentioning that the patient needs to be aware of the fact that fever resolution takes a few weeks after initiating anti-mycobacterial therapy. Keblawi at el. has shown that the number of days required for resolution of fever is variable (mean, 16 days; median, 10 days; range, 1 to 109 days) [21].

\section{Conclusions}

BCG-Osis is one of the rare and dangerous complications of intravesical BCG instillation which requires a high level of suspicion.

Although rare, urologists should keep it in the differential diagnosis when treating any patient presenting with fever after intravesical BCG instillation.

It is strongly suggested that BCG immunotherapy should be delayed for a couple of weeks when traumatic instrumentation is encountered during the procedure (if any blood came from urethra or on catheter tip).

It is imperative to send multiple urine samples for culture before starting the empirical anti-tuberculous treatment and urine sample should be sent a couple of weeks after the last dose of BCG to avoid potential false positive report from the vaccine itself, because it contains live attenuated M.Bovis. Mycobacterium Bovis isolated in this case was resistant to Pyrazinamide, reinforcing the previous recommendation of omitting it in the treatment regimen.

Treatment should not be delayed when the clinical suspicion is high. The importance of early initiation of empiric treatment of BCG-Osis cannot be overemphasized. Moreover, it may take a few weeks for the fever to settle despite being on anti-tuberculous mediation.

\section{Abbreviations}

BCG: Bacillus Calmette-Guérin; CT: Computed tomography; KUB: KidneyUreter-Bladder; EAU: European Association of Urology; NMIBC: Non-muscleinvasive bladder cancer; CIS: Carcinoma in situ; CFU: Colony-forming unit; CEA: Carcinoembryonic antigen; TB: Tuberculosis; Bpm: Beats per minute; WBC: White blood cells; CRP: C reactive protein; UTI: Urinary tract infection; AFB: Acid fast bacilli; ATT: Anti-tuberculous treatment; OD: Once daily; H\&E: Haematoxylin and Eosin; ZN: Ziehl-Neelsen; PAS: Periodic acid schiff; MDT: Multidisciplinary team; FUO: Fever-of-unknown-origin; BAL: Bronchoalveolar lavage; PCR: Polymerase chain reaction; M.Bovis: Mycobacterium Bovis.

\section{Authors' contributions}

A.G. is the author who formulated the final manuscript, reviewed the recent literature, and sent the publication. T.Q. is the author from the respiratory team who contributed to the history and physical examination, data collection, clinical follow-up and patient care during admission and after discharge, and also in manuscript writing. SL is the respiratory consultant under whom the patient was under, contributed to the clinical diagnosis and follow-up of the case. J.S. is the pathology consultant who was responsible for the laboratory and pathology investigations of the patient. S.M. is the author who had the idea of publishing the case from the start, the main urologist whom the patient was under since admission, reviewed the manuscript and approved the final manuscript. All authors have read and approved the manuscript. 


\section{Funding}

This research received no specific grant from any funding agency in the public, commercial, or not-for-profit sectors. None of the authors have any financial support or relationships that may pose a conflict of interest.

\section{Availability of data and materials}

The datasets generated during and/or analysed during the current study are available from the corresponding author on reasonable request.

\section{Declarations}

Ethics approval and consent to participate

Approval was taken from the ethical committee of the trust and consent for participation was obtained from the patient.

\section{Consent for publication}

Written informed consent for publication of the patient's clinical details and clinical images was obtained from the patient. It can be requested at any time.

\section{Competing interests}

The authors declare that they have no competing interests.

\section{Author details}

${ }^{1}$ Department of Urology and Nephrology, Darent Valley Hospital, Dartford, UK. ${ }^{2}$ Department of Respiratory Medicine, Darent Valley Hospital, Dartford, UK.

${ }^{3}$ Department of Pathology, Maidstone Hospital, Maidstone, UK. ${ }^{4}$ Department of Urology, Faculty of Medicine, Menoufia University, Al Minufiyah, Egypt.

Received: 3 May 2021 Accepted: 24 January 2022

Published online: 14 February 2022

\section{References}

1. Brandau S, Suttmann HJB (2007) Thirty years of BCG immunotherapy for non-muscle invasive bladder cancer: A success story with room for improvement. Biomed Pharmacother 61(6):299-305

2. Lamm DL (2000) Efficacy and safety of bacille Calmette-Guerin immunotherapy in superficial bladder cancer. Clin Infect Dis 31(3):S86-\$90

3. Babjuk M et al. (2020) EAU Guidelines

4. Lukacs S et al (2013) Systemic BCG-osis as a rare side effect of intravesical BCG treatment for superficial bladder cancer. Case Rep Urol 2013:1

5. Dammert $P$ et al (2013) Fever of unknown origin and pancytopenia caused by culture-proven delayed onset disseminated bacillus Calmette-Guerin (BCG) infection after intravesical instillation. Case Rep 2013:bcr2013008949

6. González-Del Vecchio M et al (2016) Differences between a probable and proven BCG infection following intravesical instillations: 16 years experience in a tertiary care hospital. Diagn Microbiol Infect Dis 85(3):338-343

7. Lamm DL et al (1986) Complications of bacillus Calmette-Guerin immunotherapy in 1,278 patients with bladder cancer. J Urol 135(2):272-274

8. Elkabani M et al (2000) Disseminated Mycobacterium bovis after intravesicular bacillus Calmette-Guerin treatments for bladder cancer. Cancer Control 7(5):476-481

9. Asín MAP-J et al (2014) Bacillus Calmette-Guérin (BCG) infection following intravesical BCG administration as adjunctive therapy for bladder cancer: incidence, risk factors, and outcome in a single-institution series and review of the literature. Medicine $\mathbf{9 3}(17): 1$

10. Sylvester RJ et al (2003) The side effects of Bacillus Calmette-Guerin in the treatment of Ta T1 bladder cancer do not predict its efficacy: results from a European Organisation for Research and Treatment of Cancer GenitoUrinary Group Phase III Trial. Eur Urol 44(4):423-428

11. Larsen ES et al (2019) The epidemiology of bacille Calmette-Guérin infections after bladder instillation from 2002 through 2017: a nationwide retrospective cohort study. BJU Int 124(6):910-916

12. Lilburn PA et al (2020) Disseminated BCG-osis and the importance of performing post-mortem examinations. J Lung Health Dis 4(2):1

13. Rachakonda T, et al (2017) Pleural Effusion Caused by Bacillus CalmetteGuérin Immunotherapy for Bladder Cancer. In: Open forum infectious diseases. Oxford University Press
14. Tasleem AM et al (2014) A late presentation of isolated lymph node tuberculosis postintravesical BCG therapy for superficial bladder cancer: a novel case. Case Rep 2014:1

15. Mehta AR, Mehta PR, Mehta RLJCR (2012) A cough conundrum in a patient with a previous history of BCG immunotherapy for bladder cancer. Case Rep 2012:bcr2012007327

16. Salvador R et al (2007) Tuberculous epididymo-orchitis after intravesical BCG therapy for superficial bladder carcinoma: sonographic findings. J Ultrasound Med 26(5):671-674

17. Pfyffer GE, Wittwer $F(2012)$ Incubation time of mycobacterial cultures: how long is long enough to issue a final negative report to the clinician? J Clin Microbiol 50(12):4188-4189

18. Choi C-HR, Lee SO, Smith GJCR (2014) Subclinical miliary Mycobacterium bovis following BCG immunotherapy for transitional cell carcinoma of the bladder. Case Rep 2014:bcr2013201202

19. Nemeth J et al (2008) Bone marrow infection with bacillus CalmetteGuérin (BCG) after intravesical immunotherapy. Wien Klin Wochenschr 120(3):121-123

20. Siatelis A et al (2011) Detection of bacillus Galmette-Guerin (Mycobacterium bovis BCG) DNA in urine and blood specimens after intravesical immunotherapy for bladder carcinoma. J Clin Microboil 49(4):1206-1208

21. Kiblawi SS et al (1981) Fever response of patients on therapy for pulmonary tuberculosis. Am Rev Respir Dis 123(1):20-24

\section{Publisher's Note}

Springer Nature remains neutral with regard to jurisdictional claims in published maps and institutional affiliations.

\section{Submit your manuscript to a SpringerOpen ${ }^{\circ}$ journal and benefit from:}

- Convenient online submission

- Rigorous peer review

- Open access: articles freely available online

- High visibility within the field

- Retaining the copyright to your article

Submit your next manuscript at $\boldsymbol{\nabla}$ springeropen.com 